\section{Indicadores demográficos e socioeconômicos associados à incapacidade funcional em idosos}

\author{
Socioeconomic and demographic indicators \\ associated with functional disability in the elderly
}

Gustavo Nunes Pereira 1

Gisele Alsina Nader Bastos 2

Giovâni Firpo Del Duca ${ }^{3}$

Ângelo José Gonçalves Bós 1

\footnotetext{
${ }^{1}$ Instituto de Geriatria e Gerontologia, Pontifícia Universidade Católica do Rio Grande do Sul, Porto Alegre, Brasil.

2 Hospital Moinhos de Vento, Universidade Federal de Ciências da Saúde de Porto Alegre, Porto Alegre, Brasil. 3 Universidade Federal de Santa Catarina, Florianópolis, Brasil.

Correspondência G. N. Pereira

Instituto de Geriatria e Gerontologia, Pontifícia Universidade Católica do Rio Grande do Sul.

Av. Ipiranga 6690, 3o andar, Porto Alegre, RS 90610-000, Brasil.

gustavojaguarao@hotmail.com
}

\section{Abstract}

This study analyzed the prevalence of functional disability in the elderly and its association with socioeconomic and demographic factors. This was a population-based cross-sectional study with a sample of 631 elders ( $\geq 60$ years). The Katz index and Lawton scale, respectively, were used to evaluate basic and instrumental activities of daily living. Demographic and socioeconomic variables were analyzed. Numerical variables were tested with the Student t test and non-numerical ones with the chi-square test. The odds of functional disability were measured by logistic regression. Prevalence rates of functional disability for basic and instrumental activities of daily living were $15.5 \%$ and $26.1 \%$, respectively. Instrumental activities presented more variables significantly associated with the outcome in the final logistic regression model. Prevalence of functional disability was not higher than described in the Brazilian literature. An important association was found between the outcome and independent variables.

Aged; Activities of Daily Living; Personal Autonomy; Socioeconomic Factors; Cross-Sectional Study

\section{Introdução}

O Brasil apresenta acelerado processo de envelhecimento, com transformações importantes na composição etária de sua população. Segundo o Censo Demográfico de 2010 (http://www.ibge. gov.br), a população de 60 anos ou mais de idade era de 20,6 milhões de pessoas contra $14,5 \mathrm{mi}$ lhões em 2000 e 10,7 milhões em 1991. O peso relativo da população idosa no início da década de 90 era de $7,3 \%$ contra $8,6 \%$ em 2000 e 10,8\% em 2010 1. O aumento da expectativa de vida não acontece de forma equitativa nos diferentes contextos socioeconômicos, assim como pessoas de diferentes níveis socioeconômicos apresentam diferentes características clínicas, entre elas a prevalência e incidência da incapacidade funcional 2,3.

Verbrugge \& Jette 4 definiram a incapacidade funcional como a dificuldade experimentada em realizar atividades em qualquer domínio da vida devido a um problema físico ou de saúde, com impactos sobre a habilidade para exercer papéis e atividades na sociedade 5 . Essas atividades são agrupadas em atividades básicas de vida diária, as quais caracterizam a habilidade para realizar tarefas de autocuidado; e em atividades instrumentais de vida diária, que incluem as tarefas que permitem a vida em comunidade 3 .

A avaliação funcional de uma população é importante na detecção de fatores de risco modificáveis, relacionados ao desempenho funcio- 
nal 5,6. Estudos que avaliam a capacidade funcional de idosos evidenciaram que a perda da funcionalidade, avaliada por meio de índices de atividades básicas e atividades instrumentais da vida diária, aumenta o risco de mortalidade em idosos 7,8,9. A capacidade funcional é a medida que melhor detecta o quadro de dependência, sendo mais eficiente do que instrumentos anteriores que relacionavam o grau de dependência com o número de comorbidades que o indivíduo apresentava 5,10,11.

Alguns fatores afetam fortemente a capacidade funcional e estão relacionados com a presença de algumas doenças e deficiências. Entretanto, a capacidade funcional também pode ser influenciada por fatores demográficos e socioeconômicos 6 .

Desse modo, torna-se imprescindível a avaliação do grau de dependência de idosos para atividades básicas e instrumentais da vida diária e a detecção de fatores socioeconômicos e demográficos que estejam associados com este desfecho.

Nesse sentido, o presente estudo objetivou estimar a prevalência de incapacidade funcional para atividades básicas e instrumentais da vida diária, assim como investigar aspectos socioeconômicos e demográficos associados em idosos residentes numa comunidade de baixa renda de Porto Alegre, Rio Grande do Sul.

\section{Métodos}

Este trabalho é parte de um amplo inquérito epidemiológico realizado nos Distritos Sanitários da Restinga e do Extremo-sul, em Porto Alegre, Brasil. A Associação Hospitalar Moinhos de Vento, em parceria com o Ministério da Saúde, realizou um inquérito epidemiológico para descrever as características demográficas e socioeconômicas dessa população e avaliar a saúde da comunidade, o acesso e o uso dos serviços médicos e odontológicos.

Os bairros avaliados no estudo foram criados na década de 70, a partir de um projeto habitacional, o qual transferiu a população economicamente desfavorável de áreas em desenvolvimento urbano em Porto Alegre para cerca de 22 quilômetros de distância do centro da cidade.

A pesquisa teve delineamento transversal de base populacional. No processo amostral por conglomerados foram definidos dois estágios: os setores censitários, definidos como unidades amostrais primárias; e os domicílios, constituindo as unidades amostrais secundárias. Foi realizado o mapeamento prévio dos 121 setores censitários existentes, constatando-se que, destes, 117 eram setores domiciliares. Na fase de identificação de domicílios foram encontrados 32.067, destes 29.929 eram habitados, 990 eram exclusivamente comerciais e 1.148 estavam desabitados.

A partir de pulos sistemáticos, fizeram parte da amostra deste trabalho todos os indivíduos com idade superior ou igual a 60 anos, residentes nos domicílios sorteados dos 117 setores. Perdas e recusas foram definidas após tentativas para realização da entrevista em no mínimo três visitas, efetuadas em dias e horários distintos.

O cálculo amostral para este estudo empregou os seguintes parâmetros e estimativas: prevalência de incapacidade funcional para atividades básicas da vida diária de $15 \%$, e $28 \%$ para atividades instrumentais, ambas com intervalo de 95\% de confiança (IC95\%) e erro aceitável de três a quatro pontos porcentuais, respectivamente. Foi utilizado um adicional de $10 \%$ para perdas e recusas e de $15 \%$ para fatores de confusão, resultando em 554 sujeitos para atividades básicas e 556 para atividades instrumentais. No entanto, outros cálculos amostrais foram realizados com intuito de se adquirir uma amostra que viabilizasse os diversos desfechos estudados no grande trabalho. Sendo assim, dentre todos os valores calculados, prevaleceu o maior tamanho amostral, que correspondeu à necessidade de entrevistar todos os idosos residentes em 1.750 domicílios.

Foram incluídos no estudo idosos detectados por visita domiciliar, que aceitaram responder ao questionário de avaliação após assinarem o Termo de Consentimento Livre e Esclarecido. Foram excluídos do estudo os idosos institucionalizados, incluindo aqueles que residem em Instituições de Longa Permanência (ILPIs).

Os entrevistadores receberam um treinamento que totalizou 80 horas, divididas em duas semanas. O trabalho foi divulgado por carta de apresentação, rádios locais, jornais e cartazes, e o campo foi realizado no período de julho a dezembro de 2009. Foi realizado um controle de qualidade em $10 \%$ da amostra via telefone e os dados foram checados por um programa denominado Office Remark (Gravic Inc., Filadélfia, Estados Unidos). Além disso, as inconsistências do banco de dados foram verificadas.

Os desfechos incapacidade funcional para atividades básicas e instrumentais da vida diária foram avaliados, respectivamente, pelo índice de Katz 12 e pela escala de Lawton 13 . Foram investigadas seis atividades básicas (banhar-se, vestirse, ir ao banheiro, comer, deitar-se e levantar-se da cama/cadeira e ser continente nas funções de urinar/evacuar) e oito atividades instrumentais (usar o telefone, deslocar-se utilizando meio de 
transporte, fazer compras, preparar a refeição, arrumar a casa, lavar a roupa, cuidar de finanças e tomar remédios). Tanto a incapacidade funcional para as atividades básicas quanto para as atividades instrumentais foram definidas da mesma forma: necessidade de ajuda parcial ou total para, no mínimo, uma das atividades diárias investigadas. Assim, os índices de Katz e Lawton foram dicotomizados em: zero (independente para todas as atividades) e um (dependente para uma ou mais atividades).

Os aspectos demográficos e socioeconômicos analisados foram: faixa etária por década de vida (60-69, 70-79, 80-89 e 90 anos ou mais), sexo, número de moradores que residem na casa do idoso entrevistado, escolaridade (categorizada em: nunca estudou, um a quatro, cinco a oito e nove ou mais anos completos de estudos), situação conjugal [classificada em: casado(a), viúvo(a) e outros], nível econômico (divisão em cinco classes sociais segundo questionário da Associação Brasileira de Empresas e Pesquisa (ABEP), e classificados em: classes A-B, classe C e classes D-E) e presença de cuidador (sim ou não).

As variáveis média de idade e número de moradores por casa foram avaliadas para cada desfecho e comparadas pelo teste t de Student. Tabelas de distribuição foram construídas cruzando desfecho e variáveis não numéricas, e testadas pelo teste do qui-quadrado.

O risco para o desfecho de incapacidade funcional foi medido pela regressão logística e expresso em razão de chance com IC95\%. Ambos os intervalos de confiança acima de um foram considerados significativamente risco para o desfecho e, quando abaixo de um, foram considerados fatores de proteção para o desfecho.

Em virtude da metodologia utilizada com amostragem sistemática e probabilidade ao tamanho do setor censitário, além do baixo porcentual de perdas e recusas, os dados obtidos poderão ser generalizados para todos os idosos residentes em bairros de população com vulnerabilidade social.

$\mathrm{O}$ projeto de pesquisa foi aprovado pelo Comitê de Ética do Hospital Moinhos de Vento, conforme o Protocolo 2009/28, e pelo Comitê de Ética em Pesquisa da Pontifícia Universidade Católica do Rio Grande do Sul (PUC-RS), sob o registro 10/05205. Este trabalho cumpriu integralmente os princípios éticos contidos na Declaração de Helsinki (2000), além do atendimento à legislação específica deste país.

\section{Resultados}

A amostra compreendeu $13,4 \%$ dos moradores idosos dos bairros de atuação da pesquisa, que totalizariam 671 idosos. Quarenta moradores foram considerados como perda e recusa, totalizando 6\%. Assim, foram entrevistados 631 idosos.

A distribuição dos idosos conforme as variáveis independentes é apresentada na Tabela 1.

O sexo feminino foi predominante, totalizando $59,7 \%$ da amostra. A faixa etária amostral variou de 60-96 anos, com idade média de 68,5 anos $( \pm 7,1)$. Em relação à distribuição por década de vida, verificou-se que 398 (63\%) idosos tinham entre 60 e 69 anos e apenas $54(8,6 \%)$ tinham mais de 80 anos. Foi verificado que 100 $(15,8 \%)$ idosos moravam sozinhos, $329(52,1 \%)$ eram casados, 183 (29\%) eram viúvos e 161 (25,5\%) contavam com o auxílio de um cuidador. Após avaliar o nível econômico dos indivíduos residentes nas comunidades do estudo, segundo o questionário da ABEP com cinco diferentes categorias, 186 (29,5\%) idosos foram classificados como pertencentes às classes A e B, $337(53,4 \%)$ à classe $\mathrm{C}$ e $108(17,1 \%)$ moradores foram categorizados como integrantes das classes D e E. Já quando observamos a escolaridade em anos de estudos, 123 (19,5\%) referiram nunca ter estudado, 186 (29,5\%) idosos referiram ter estudado de um a quatro anos, $219(34,7 \%)$ de cinco a oito e 103 (16,3\%) entrevistados disseram ter concluído nove ou mais anos de estudos.

Quando observada a prevalência de incapacidade funcional, constatou-se que 165 (26,1\%) idosos necessitavam de auxílio para realizar no mínimo uma atividade instrumental da vida diária e $98(15,5 \%)$ para atividades básicas.

Em relação ao gênero dos idosos, verificou-se que $29(11,4 \%)$ homens e 69 (18,3\%) mulheres apresentavam dificuldade para realizar ao menos uma atividade básica. Essa diferença nas frequências foi significativa ( $p=0,019)$. Ao analisarmos as atividades instrumentais, constatou-se que $57(22,4 \%)$ homens e 108 (28,6\%) mulheres apresentavam dependência para realizar ao menos uma atividade instrumental. Essa diferença não foi significativa $(\mathrm{p}=0,081)$.

As frequências das variáveis independentes escolaridade, faixa etária, sexo, situação conjugal e presença de cuidador foram significativamente associadas com o desfecho incapacidade para atividades básicas.

Nesta análise, os idosos que nunca estudaram apresentaram a maior frequência de incapacidade $(p=0,010)$, sendo esta associação linear, pois maiores níveis de escolaridade apresentaram menores frequências do desfecho. Esse 
Distribuição dos idosos conforme as variáveis independentes e suas frequências para aqueles que apresentam incapacidade funcional para atividades básicas e instrumentais. Rio Grande do Sul, Brasil, 2009.

\begin{tabular}{|c|c|c|c|c|c|c|c|c|}
\hline & \multirow{2}{*}{\multicolumn{2}{|c|}{ Total }} & \multicolumn{6}{|c|}{ Incapacidade } \\
\hline & & & \multicolumn{3}{|c|}{ ABVD } & \multicolumn{3}{|c|}{ AIVD } \\
\hline & $\mathrm{n}$ & $\%$ & $\mathrm{n}$ & $\%$ & Valor de $p$ & $\mathrm{n}$ & $\%$ & Valor de $p$ \\
\hline ABEP & & & & & 0,419 & & & 0,980 \\
\hline$A-B$ & 186 & 29,5 & 34 & 18,3 & & 48 & 25,8 & \\
\hline C & 337 & 53,4 & 50 & 14,8 & & 88 & 26,1 & \\
\hline$D-E$ & 108 & 17,1 & 14 & 13,0 & & 29 & 26,9 & \\
\hline Escolaridade & & & & & 0,010 * & & & $<0,001$ * \\
\hline Nunca estudou & 123 & 19,5 & 31 & 25,2 & & 66 & 53,7 & \\
\hline $1-4$ anos & 186 & 29,5 & 27 & 14,5 & & 44 & 23,7 & \\
\hline $5-8$ anos & 219 & 34,7 & 27 & 12,3 & & 43 & 19,6 & \\
\hline 9 anos ou mais & 103 & 16,3 & 13 & 12,6 & & 12 & 11,7 & \\
\hline Faixa etária (anos) & & & & & $<0,001$ * & & & $<0,001 *$ \\
\hline $60-69$ & 398 & 63,0 & 41 & 10,3 & & 67 & 16,8 & \\
\hline $70-79$ & 179 & 28,4 & 36 & 20,1 & & 64 & 35,8 & \\
\hline 80 ou mais & 54 & 8,6 & 21 & 38,9 & & 34 & 63,0 & \\
\hline Morar sozinho & & & & & 0,960 & & & 0,284 \\
\hline Sim & 100 & 15,8 & 16 & 16,0 & & 24 & 24,0 & \\
\hline Não & 531 & 84,2 & 82 & 15,4 & & 141 & 26,6 & \\
\hline Sexo & & & & & 0,019 * & & & 0,081 \\
\hline Masculino & 254 & 40,3 & 29 & 11,4 & & 57 & 22,4 & \\
\hline Feminino & 377 & 59,7 & 69 & 18,3 & & 108 & 28,6 & \\
\hline Estado civil & & & & & 0,001 * & & & $<0,001$ * \\
\hline Casado & 329 & 52,1 & 37 & 11,2 & & 55 & 16,7 & \\
\hline Viúvo & 183 & 29,0 & 43 & 23,5 & & 33 & 27,7 & \\
\hline Outros & 119 & 18,9 & 18 & 15,1 & & 77 & 42,1 & \\
\hline Presença de cuidador & & & & & $<0,001 *$ & & & $<0,001 *$ \\
\hline Sim & 161 & 25,5 & 63 & 39,1 & & 99 & 61,5 & \\
\hline Não & 470 & 74,5 & 35 & 7,4 & & 66 & 14,0 & \\
\hline
\end{tabular}

ABEP: Associação Brasileira de Empresas e Pesquisa (nível econômico segundo os critérios da ABEP); ABVD: atividades básicas de vida diária; AIVD: atividades instrumentais de vida diária.

* Valores significativos.

mesmo efeito foi observado na variável faixa etária, na qual idosos mais velhos apresentaram maior incapacidade $(\mathrm{p}<0,001)$, uma vez que estas prevalências quase dobraram a cada década de vida. Mulheres $(\mathrm{p}=0,019)$ e viúvas(os) $(\mathrm{p}=0,001)$ apresentaram maiores índices de dependência. Uma contradição foi observada entre as variáveis presença de cuidador e morar sozinho, pois a porcentagem de idosos com incapacidade foi evidentemente maior nos que possuíam cuidador, mas praticamente igual nos idosos que moravam ou não sozinhos.

Ao realizar a comparação das mesmas frequências com o desfecho para atividades instrumentais, verificou-se associação significativa com escolaridade, faixa etária, situação conjugal e presença de cuidador. A relação também foi linear para escolaridade e faixa etária $(\mathrm{p}<0,001)$, porém com um percentual mais elevado, principalmente em idosos que nunca estudaram e os que têm acima de oitenta anos. Os idosos que não moram sozinhos apresentaram menores índices de dependência, embora esta associação não tenha sido significativa. A maioria dos idosos que possuía cuidador apresentou dependência para atividades instrumentais ( $p<0,001)$. Idosos(as) viúvos(as) apresentaram maiores índices de incapacidade funcional $(\mathrm{p}<0,001)$.

A variável presença de cuidador não foi incluída nos modelos da regressão logística para atividades básicas e instrumentais, pois não pode ser interpretada como independente. Os mode- 
los finais ajustados de regressão logística foram alcançados usando-se a metodologia de retirada sistemática das variáveis menos significativas, a partir do modelo completo que incluía todas as variáveis independentes do estudo. Dessa maneira, para atividades básicas mantiveram-se significativas as variáveis da Tabela 2 e, para atividades instrumentais as da Tabela 3 .

A Tabela 2 apresenta o modelo final de regressão logística com as variáveis que mantiveram-se significativas para o desfecho incapacidade funcional em atividades básicas.

Idosos pertencentes às classes sociais D-E, segundo o questionário da ABEP, apresentaram uma chance $56 \%$ menor de desenvolver incapacidade funcional quando comparados a idosos das classes A-B ( $p=0,03)$. Inversamente ao nível econômico, quanto maior a escolaridade menores foram as chances de apresentar o desfecho.

As chances de desenvolver o desfecho dobraram na comparação por década de vida entre as categorias de 70-79 e 60-69 anos ( $p=0,01)$, e entre 80 anos ou mais e $60-69$ anos $(\mathrm{p}<0,001)$.

A Tabela 3 apresenta o modelo final de regressão logística com as variáveis que se mantiveram significativas para o desfecho incapacidade funcional em atividades instrumentais.

Resultados semelhantes foram encontrados ao associar o desfecho em atividades instrumentais com as classes da ABEP $(p=0,01)$. Idosos com baixa escolaridade também apresentaram uma chance maior de desenvolverem incapacidade funcional, sendo estes resultados mais significativos do que os observados nas atividades básicas ( $p<0,001)$. Em modelos que não incluíam escolaridade, a variável ABEP não foi significativa.

Viúvos apresentaram chance 2,94 vezes maior de apresentar o desfecho quando comparados a casados. A comparação entre faixas etárias também foi semelhante ao modelo de regressão para atividades básicas, porém mais significativa $(p<0,001)$.

\section{Discussão}

A comparação das prevalências de incapacidade funcional para atividades básicas e instrumentais em diferentes estudos é prejudicada, pois os mesmos utilizam diferentes escalas e pontos de corte para o desfecho. Este estudo encontrou prevalências de incapacidade funcional de 15,7\% para atividades básicas e de 26,2\% para atividades instrumentais.

Esses achados são coincidentes aos encontrados por um estudo de base populacional realizado com idosos de Belo Horizonte 14 com média de idade semelhante. Utilizando-se o índice de
Tabela 2

Modelo final de regressão logística de incapacidade funcional para atividades básicas da vida diária. Rio Grande do Sul, Brasil, 2009.

\begin{tabular}{lcc}
\hline Atividades básicas da vida diária & Razão de chances & Valor de $\mathbf{p}$ \\
\hline ABEP (C/A-B) & 0,66 & 0,12 \\
ABEP (D-E/A-B) * & 0,44 & 0,03 \\
Escolaridade (1-4 anos/nunca estudou) & 0,56 & 0,06 \\
Escolaridade (5-8 anos/nunca estudou) & 0,54 & 0,06 \\
Escolaridade ( $\geq 9$ anos/nunca estudou) & 0,50 & 0,08 \\
Estado civil (outros/casado) & 1,33 & 0,39 \\
Estado civil (viúvo/casado) & 1,53 & 0,14 \\
Faixa etária (70-79 anos/60-69 anos) * & 1,92 & 0,01 \\
Faixa etária ( $\geq 80$ anos/60-69 anos) * & 3,95 & $<0,001$ \\
Sexo feminino & 1,36 & 0,24 \\
\hline
\end{tabular}

ABEP: Associação Brasileira de Empresas e Pesquisa (nível econômico segundo os critérios da ABEP)

*Valores significativos.

Tabela 3

Modelo final de regressão logística de incapacidade funcional para atividades instrumentais da vida diária. Rio Grande do Sul, Brasil, 2009.

\begin{tabular}{lcc}
\hline Atividades instrumentais da vida diária & Razão de chances & Valor de $\mathbf{p}$ \\
\hline ABEP (C/A-B) & 0,69 & 0,14 \\
ABEP (D-E/A-B) * & 0,44 & 0,01 \\
Escolaridade (1-4 anos/nunca estudou) * & 0,25 & $<0,001$ \\
Escolaridade (5-8 anos/nunca estudou) * & 0,24 & $<0,001$ \\
Escolaridade ( $\geq 9$ anos/nunca estudou) * & 0,10 & $<0,001$ \\
Estado civil (outros/casado) * & 2,36 & 0,002 \\
Estado civil (viúvo/casado) * & 2,94 & $<0,001$ \\
Faixa etária (70-79 anos/60-69 anos) * & 2,11 & $<0,001$ \\
Faixa etária ( $\geq 80$ anos/60-69 anos) * & 4,70 & $<0,001$ \\
Sexo feminino & 0,74 & 0,21 \\
\hline
\end{tabular}

ABEP: Associação Brasileira de Empresas e Pesquisa (nível econômico segundo os critérios da ABEP)

* Valores significativos.

Katz e o mesmo critério de corte deste estudo foi observada uma prevalência de $16 \%$. No entanto, em uma população geograficamente mais próxima, como a do município de Pelotas 15 , os níveis de incapacidade funcional foram de $26,8 \%$.

Em relação às atividades instrumentais, observou ainda em idosos de Pelotas uma prevalência semelhante à presente estudo, com $28,8 \%$ de incapacidade funcional, mensurada pela escala de Lawton. 
A incapacidade funcional normalmente é relacionada a aspectos de saúde da população idosa, existindo poucos estudos na literatura nacional e internacional associando o desfecho a indicadores demográficos e socioeconômicos, os quais demonstraram apresentar associação significativa, principalmente na incapacidade em atividades instrumentais.

Nosso estudo também testou a associação da incapacidade funcional tanto para atividades básicas quanto para instrumentais, com as mesmas variáveis independentes. Analisou-se, dessa forma, as semelhanças e diferenças nos dois domínios, proporcionando a comparação das chances de desenvolver o desfecho devido a alterações nas diferentes variáveis em cada situação.

A progressão da idade esteve significativamente associada à maior prevalência de incapacidade funcional, tanto para atividades básicas quanto para atividades instrumentais. Essa associação foi encontrada em outros estudos com populações semelhantes 5,15,16.

O sexo feminino também apresentou inicialmente associação significativa com maior incapacidade funcional para atividades básicas na análise individual e, após análise ajustada demonstrou indicativo de significância. Esse resultado corrobora com trabalhos 15,16,17 que apontam a influência da idade e das variáveis socioeconômicas nas diferenças observadas entre os gêneros. A maior incapacidade nas mulheres provavelmente está associada também ao fato de as mesmas apresentarem maior prevalência de condições incapacitantes não fatais e, com isto, uma sobrevida maior, tornando-se mais suscetíveis ao desfecho em questão. Ao contrário das atividades básicas, as instrumentais não foram associadas com o gênero.

Em análise complementar, observamos que o sexo masculino apresentava significativamente maior dependência para as atividades de lavar a roupa e limpar a casa, atividades estas culturalmente atribuídas ao sexo feminino. Em contraste, as mulheres apresentavam dependência significativamente mais prevalente para utilizar transporte. Variáveis socioeconômicas não foram determinantes nessa associação.

Embora a variável escolaridade tenha sido associada significativamente ao desfecho tanto em atividades básicas quanto instrumentais, esta relação foi maior com a segunda, provavelmente por apresentar maior dificuldade intelectual. Outros achados também evidenciaram maior incapacidade funcional em idosos com menor escolaridade 5,16. A aparente proteção para incapacidade funcional, tanto para atividades básicas quanto para instrumentais, determinada pelo menor nível econômico foi contrastante com o observado na escolaridade, apesar de idosos com menor escolaridade apresentarem um menor nível econômico. Um estudo realizado com 33.515 idosos 17, utilizando dados obtidos pela Pesquisa Nacional por Amostra de Domicílios (PNAD), por meio de entrevistas domiciliares, verificou que com o aumento dos níveis de escolaridade e renda diminuiu a probabilidade dos idosos desenvolverem incapacidade funcional. No entanto, a incapacidade funcional foi mensurada pela capacidade de subir uma ladeira ou escada.

Destacamos a importância de novos estudos que, assim como este, verifiquem a associação da incapacidade funcional para atividades básicas e instrumentais, utilizando escalas validadas na literatura nacional, com variáveis indicativas de nível econômico e escolaridade, de forma conjunta e controlada por outros fatores, como sexo e idade.

A avaliação segundo os critérios da ABEP foi escolhida para aferir o nível econômico, devido ao fato de os autores julgarem que os domínios avaliados remetem à forma mais indicada de aferir a estrutura familiar a que o idoso está submetido.

Em relação ao estado civil, constatou-se que idosos(as) viúvos(as) apresentaram maior incapacidade funcional para atividades básicas e instrumentais, mas após análise ajustada a relação entre a variável e o desfecho manteve-se significativa apenas para as atividades instrumentais. Outros estudos de base populacional 5,16 corroboram esses achados, mas um levantamento realizado com idosos de um plano de saúde privado evidenciou associação significativa apenas na análise bruta, apresentando perda de significância após o ajuste por idade 18. Acreditamos que isso possa ter sido influenciado pelo fato de que a situação conjugal viúvo(a) seja mais prevalente em mulheres e pessoas mais idosas.

Apesar de não ter sido significativo para incapacidade, outros trabalhos 19,20 relataram que o perfil do idoso que mora sozinho no Brasil é composto em sua maioria por mulheres viúvas com doenças crônicas. O conjunto desses fatores pode explicar os resultados encontrados em nosso estudo.

Outro trabalho relatou que a atividade física praticada nos momentos de lazer ao longo da vida e as alterações cognitivas parecem ser determinantes na classificação da incapacidade funcional, mesmo quando controlado para as variáveis sociodemográficas 21 . No entanto, o objetivo do nosso estudo foi investigar a possível associação de aspectos socioeconômicos e demográficos com a incapacidade funcional.

Podemos citar o delineamento transversal como a principal limitação do estudo, pois im- 
pede a realização de uma análise causal, impossibilitando o entendimento da temporalidade na relação entre a incapacidade funcional e as exposições do estudo. No entanto, foi realizada a análise ajustada para evitar possíveis fatores de confusão.

\section{Conclusão}

A prevalência de incapacidade funcional, tanto para atividades básicas quanto para atividades instrumentais da vida diária, não foi maior do que a descrita na literatura brasileira. Encontrouse uma importante associação do desfecho com as variáveis analisadas, principalmente na associação das variáveis demográficas e socioeconômicas com a incapacidade funcional para atividades instrumentais.

\section{Resumo}

O objetivo do estudo foi observar a frequência de incapacidade funcional de idosos e sua associação com fatores socioeconômicos e demográficos. Foi realizado um estudo transversal de base populacional, incluindo 631 idosos (60 anos ou mais). Para a avaliação das atividades básicas e instrumentais da vida diária foram empregados o indice de Katz e a escala de Lawton, respectivamente. Foram analisadas as variáveis demográficas e socioeconômicas. As variáveis numéricas foram testadas pelo t de Student e as não numéricas pelo qui-quadrado. A chance de apresentar incapacidade funcional foi medida pela regressão logística. As prevalências de incapacidade funcional para as atividades básicas e instrumentais da vida diária foram de $15,5 \%$ e 26,1\%, respectivamente. As atividades instrumentais apresentaram maior número de variáveis significativamente associadas ao desfecho no modelo final de regressão logística. A prevalência de incapacidade funcional não foi maior do que a descrita na literatura brasileira. Encontrou-se uma importante associação do desfecho com as variáveis analisadas.

Idoso; Atividades Cotidianas; Autonomia Pessoal; Fatores Socioeconômicos; Estudos Transversais

\section{Colaboradores}

G. N. Pereira participou da análise estatística e interpretação dos dados, além da redação do manuscrito. G. A. N. Bastos participou da concepção e desenho do estudo por meio da elaboração do projeto de pesquisa e treinamento dos entrevistadores. Coordenou a coleta e limpeza do banco de dados e contribuiu no manuscrito com a revisão crítica do mesmo. G. F. Del Duca participou da concepção do manuscrito, elaborou o instrumento de pesquisa, auxiliou na revisão da literatura e análise dos dados, e revisou criticamente o manuscrito. A. J. G. Bós orientou a análise estatística e sua interpretação, revisou a metodologia e o manuscrito. Todos os autores acima citados revisaram o texto em suas versões inicial e final.

\section{Agradecimentos}

Pesquisa realizada no âmbito do projeto Desenvolvimento de Técnicas de Operação e Gestão de Serviços de Saúde em uma Região Intramunicipal de Porto Alegre - Distritos Sanitários da Restinga e Extremo-sul, de acordo com o Programa de Apoio ao Desenvolvimento Institucional do Sistema Único de Saúde (PROADISUS), firmado entre o Ministério da Saúde e a Associação Hospitalar Moinhos de Vento, por meio do termo de ajuste de número 06/2008, assinado em 17 de novembro de 2008 . 


\section{Referências}

1. Instituto Brasileiro de Geografia e Estatística. Perfil dos idosos responsáveis pelos domicílios no Brasil. 2000. http://www.ibge.gov.br/home/estatistica/ populacao/perfilidoso/perfidosos2000.pdf (acessado em 14/Fev/2011).

2. Freitas EV, Py L, Gorzoni ML, Cançado FAX. Tratado de geriatria e gerontologia. Rio de Janeiro: Editora Guanabara Koogan; 2002.

3. Lollar DJ, Crews JE. Redefining the role of public health in disability. Annu Rev Public Health 2003; 24:195-208.

4. Verbrugge LM, Jette AM. The disablement process. Soc Sci Med 1994; 38:1-14.

5. Rosa TEC, Benício MHD'A, Latorre MRDO, Ramos LR. Fatores determinantes da capacidade funcional entre idoso. Rev Saúde Pública 2003; 37:40-8.

6. Costa EC, Nakatani AYK, Bachioni MM. Capacidade de idosos da comunidade para desenvolver atividades de vida diária e atividades instrumentais de vida diária. Acta Paul Enferm 2006; 19:35-43.

7. Pavarini SCI, Neri AL. Compreendendo dependência, independência e autonomia no contexto domiciliar: conceitos, atitudes e comportamentos São Paulo: Editora Atheneu; 2000.

8. Maciel ACC, Guerra RO. Limitação funcional e sobrevida em idosos de comunidade. Rev Assoc Med Bras 2008; 54:347-52.

9. Larsen KS, Kirsten A. Tiredness in daily activities: a subjective measure for the identification of frailty among non-disabled community-living older adults. Arch Gerontol Geriatr 2007; 44:83-93.

10. Bonardi G, Azevedo e Souza VB, Moraes JFD. Incapacidade funcional e idosos: um desafio para os profissionais de saúde. Sci Med 2007; 17:138-44.

11. Parahyba MI, Simões CCS. A prevalência de incapacidade funcional em idosos no Brasil. Ciênc Saúde Coletiva 2006; 11:967-74.

12. Katz S, Ford AB, Moskowitz RW, Jackson BA, Jaffe MW. Studies of illness in the aged. the index of ADL: a standardized measure of biological and psychosocial function. JAMA 1963; 185:914-9.
13. Lawton MP, Brody EM. Assessment of older people: self-maintaining and instrumental activities of daily living. Gerontologist 1969; 9:179-86.

14. Giacomin K, Peixoto S, Uchoa E, Lima-Costa M. Estudo de base populacional dos fatores associados à incapacidade funcional entre idosos na região metropolitana de Belo Horizonte, Minas Gerais, Brasil. Cad Saúde Pública 2008; 24:1260-70.

15. Del Duca GF, Silva MC, Hallal PC. Incapacidade funcional para atividades básicas e instrumentais de vida diária em idosos. Rev Saúde Pública 2009; 43:796-805.

16. Santos KA, Koszuoski R, Dias-da-costa JS, Pattussi MP. Fatores associados com a incapacidade funcional em idosos do Município de Guatambu, Santa Catarina, Brasil. Cad Saúde Pública 2007; 23:2781-8.

17. Alves LC, Leite IC, Machado CJ. Fatores associados à incapacidade funcional dos idosos no Brasil: análise multinível. Rev Saúde Pública 2010; 44: 468-78.

18. Cardoso JH, Costa JSD. Características epidemiológicas, capacidade funcional e fatores associados em idosos de um plano de saúde. Ciênc Saúde Coletiva 2010; 15:2871-8.

19. Maciel ACC, Guerra RO. Influência dos fatores biopsicossociais sobre a capacidade funcional dos idosos residentes no nordeste do Brasil. Rev Bras Epidemiol 2007; 10:178-89.

20. Ramos LR. Fatores determinantes do envelhecimento saudável em idosos residentes em centro urbano: Projeto Epidoso, São Paulo. Cad Saúde Pública 2003; 19:793-7.

21. Virtuoso Júnior JS, Guerra RO. Incapacidade funcional em mulheres idosas de baixa renda. Ciênc Saúde Coletiva 2011; 16:2541-7.

Recebido em 14/Mar/2012

Versão final reapresentada em 17/Jul/2012

Aprovado em 07/Ago/2012 